\title{
Intervenção com pais: Impactos em curto e longo prazo no comportamento infantil'
}

\author{
Intervention with parents: Short- and long-term impacts on child behavior
}

Fabiana Cia ${ }^{[a]}$ Elizabeth Joan Barham ${ }^{[b]}$

\footnotetext{
[a] Psicóloga, doutora em Educação Especial pela Universidade Federal de São Carlos (UFSCar), professora adjunta do curso de Licenciatura em Educação Especial da Universidade Federal de São Carlos/UFSCar), São Carlos, SP - Brasil, e-mail: fabianacia@ufscar.br

${ }^{[b]}$ Psicóloga, doutora em Psicologia Social e de Desenvolvimento Aplicado pela University of Guelph, Canadá, professora associada do Departamento de Psicologia e do Programa de Pós-Graduação em Psicologia da Universidade Federal de São Carlos (UFSCar), São Carlos, SP Brasil, e-mail: lisa@ufscar.br
}

Recebido: $15 / 02 / 2011$ Received: 02/15/2011

Aprovado: $17 / 03 / 2011$ Approved: 03/17/2011

\section{Resumo}

Este estudo avaliou o impacto de um programa de intervenção educativa com pais sobre os problemas de comportamento das crianças. Os participantes foram divididos em três grupos: (a) GE1 - 29 pais/mães, apenas os pais receberam intervenção; (b) GE2 - 36 pais/mães, apenas as mães receberam intervenção; e GC - 34 pais/mães que não receberam intervenção. Pais e mães avaliaram os comportamentos dos filhos no pré-teste, pós-teste e follow-up. Ambos os pais indicaram diminuição nos problemas de comportamento externalizantes e total na fase de pós-teste para GE1 e GE2; e as avaliações dos pais também indicaram menor índice de problemas de comportamento internalizantes no pós-teste para GE1. Ao comparar pós-teste e follow-up, as avaliações das mães indicaram menores índices de problemas de comportamento externalizantes e total no follow-up para GE1 e GE2.

Palavras-chave: Programa de intervenção. Pais. Problemas de comportamento. Prevenção.

\begin{abstract}
This study assessed short- and long-term impacts of an intervention education program for parents on child behavior problems. The participants were divided into three groups: (a) EG1 - 29 fathers/mothers, only fathers received intervention; (b) EG2 - 36 fathers/mothers, only mothers received intervention; and (c) GC - 34 fathers/mothers, parents took no part in the intervention program. Parents evaluated their child's behavior in pretest, posttest and follow-up. Mothers' and fathers' assessments indicated lower rates of externalizing and total behavior problems in posttest for EG1and EG2; and father's assessment also indicated lower rates of internalizing problems in posttest for EG1. In comparing posttest and follow-up results (long-term impact), mothers' assessments indicated lower rates of externalizing and total problems in the follow-up for both experimental groups.
\end{abstract}

Keywords: Intervention program. Parents. Behavior problems. Prevention.

\footnotetext{
1 Este trabalho é derivado da tese de doutorado da primeira autora (com apoio financeiro da Fapesp).
} 
Este estudo aborda a temática do desenvolvimento infantil com base na teoria bioecológica de Bronfenbrenner, considerando os impactos que um programa de intervenção para pais têm sobre os problemas de comportamento internalizantes e externalizantes das crianças. Assim, a presente revisão está dividida em dois tópicos: (1) teoria bioecológica de Bronfenbrenner e (2) programas de intervenção com pais.

\section{Teoria Bioecológica de Bronfenbrenner}

Para melhor identificar diferentes fontes de risco para o desenvolvimento infantil e as ligações entre eles, faz-se necessário considerar uma perspectiva teórica que une o envolvimento parental com diferentes aspectos do desenvolvimento social da criança. Nesse sentido, uma perspectiva teórica importante para essa pesquisa é a Teoria de Sistemas Ecológicos de Urie Bronfenbrenner. Segundo Bronfenbrenner, "o desenvolvimento humano envolve a acomodação progressiva, mútua, entre um ser humano (nesse caso, a criança) e os elementos mutantes dos ambientes em que convive" (Bronfenbrenner, 1996, p. 18). O desenvolvimento humano pode ser estimulado ou inibido pelas interações com diferentes pessoas, que desempenham papéis diferenciados, e pelo engajamento e participação nas atividades em diferentes espaços. Um ambiente de especial importância para a criança é o familiar, no qual, por exemplo, o pai interage com ela, de forma que cada um influencia no outro (Bronfenbrenner, 1999).

0 processo de desenvolvimento também é afetado pelas relações entre diferentes ambientes em que a criança convive (por exemplo, o escolar e o familiar), e pelos contextos socioculturais mais amplos em que estão inseridos. Nesse sentido, para potencializar o desenvolvimento social de uma criança, não se deve considerar apenas o ambiente imediato do indivíduo (por exemplo, apenas a escola ou a família) como influenciador no processo de desenvolvimento; é necessário considerar também as interconexões entre esses dois ambientes imediatos e outras influências indiretas (como o trabalho dos pais), além das influências externas advindas de contextos socioculturais (como a abertura cultural para o envolvimento paterno direto). Bronfenbrenner (1999) ainda defende a ideia de que aquilo que importa para o comportamento e para o desenvolvimento humano é a percepção das pessoas sobre o ambiente, uma vez que a cultura e a história de vida individual influenciam constantemente na maneira como o entendemos.

Segundo a perspectiva bioecológica de Bronfenbrenner, deve-se interpretar o desenvolvimento humano considerando a interação entre quatro núcleos: o processo, a pessoa, o contexto e o tempo. 0 processo é o principal mecanismo responsável pelo desenvolvimento, que se dá por meio de interações recíprocas progressivamente mais complexas, envolvendo um organismo biopsicologicamente em evolução e as pessoas, os objetos e os símbolos do ambiente imediato. Para ser eficaz, deve ocorrer em uma base regular por um período extensivo de tempo. Os processos proximais são atividades do dia a dia nas quais as pessoas se engajam; essas atividades são influenciadas pelas características da pessoa em desenvolvimento, pelos contextos imediatos e remotos, pela intensidade e frequência do período de tempo em que o indivíduo foi exposto ao processo proximal e o ambiente em que ocorreu, sendo que tais aspectos sofrem influências dos fatores genéticos (Narvaz \& Koller, 2004).

A pessoa é constituída tanto de características determinadas biopsicologicamente, quanto de características construídas na sua interação com o meio, considerando as crenças, os valores, os traços da personalidade etc. Desse modo, existe uma relação funcional e bidirecional entre as características da pessoa e do seu ambiente.

0 terceiro componente é o contexto, que diz respeito às inter-relações entre microssistema, mesossistema, exossistema e macrossistema) (Bronfenbrenner, 1996). Como quarto componente tem-se o tempo (Narvaz \& Koller, 2004), que é analisado em três níveis: microtempo - continuidade e descontinuidade dentro de pequenas partes dos processos proximais, cuja efetividade dependerá da ocorrência de uma interação recíproca, mais complexa e transcorrida em um tempo regular; mesotempo - periodicidade dos processos proximais em intervalos maiores com efeitos cumulativos que trarão benefícios ao desenvolvimento; e macrotempo - crenças, expectativas e mudanças na sociedade, através das gerações, assim como a inter-relação desses eventos com os resultados do desenvolvimento, no ciclo vital. Devem-se considerar, 
para analisar o tempo, as mudanças das pessoas, ambientes, assim como as da interação entre estes.

Considerando a perspectiva Bioecológica de Bronfenbrenner, pode-se supor que a estimulação que uma criança vivencia ao longo da sua vida principalmente no ambiente familiar (que foi o foco de intervenção deste estudo) - pode vir a influenciar (positivamente ou negativamente) no curso do seu desenvolvimento, mais especificamente no emocional, social e, por consequência, na sua aprendizagem.

0 enfoque da intervenção realizada no presente estudo foi na qualidade da interação entre pais e filhos (ao nível microssistêmico), abordando temáticas para a maximização do desenvolvimento infantil, práticas parentais, estresse, conflitos, entre outros. Além disso, o mesossistema foi priorizado, porque o programa se refere às relações estabelecidas entre a escola e a família da criança. No nível exossistêmico, recorreu-se aos recursos sociais, que poderiam oferecer apoio às famílias; isso porque, mesmo não estando diretamente relacionados à criança, poderiam influenciar sua vivência; um exemplo seria situação em que os pais participassem de um programa de intervenção ou empregassem estratégias de enfrentamento de estresse no trabalho, podendo refletir positivamente no seu relacionamento com os filhos. Por fim, a realização deste estudo foi possível pelas mudanças, ainda graduais, da formação de identidade dos homens, que não está voltada apenas para ser o provedor financeiro, mas também para participar mais ativamente da vida familiar, inclusive da educação de sua prole, ao nível macrossistêmico.

\section{Programa de intervenção com pais}

Neste estudo adotou-se a definição de problemas comportamentais e emocionais, classificados em dois grandes grupos: os externalizantes (que são expressos predominantemente em relação a outras pessoas) e os internalizantes (que são expressos predominantemente em relação ao próprio indivíduo). Os problemas externalizantes são mais frequentes em transtornos que envolvem agressividade física ou verbal, comportamentos opositores ou desafiadores, condutas antissociais (mentir e roubar) e comportamentos de risco (como uso de substâncias psicoativas). Os problemas internalizantes são mais prontamente identificáveis em transtornos como depressão, isolamento social, ansiedade e fobia social (Del Prette A. \& Del Prette Z., 2005; Gresham \& Elliott, 1990).

Para Pacheco, Alvarenga, Reppold, Piccinini, e Hutz (2005), os problemas de comportamento entre as crianças estão associados, principalmente, às características das interações familiares, à medida que os membros da família treinam diretamente esse padrão comportamental na criança. Conte (2001) enumera vários comportamentos dos pais que podem levar ao desenvolvimento de problemas de comportamento por parte dos filhos, dividindo esses comportamentos em dois blocos: (a) ações que os pais têm diante de seu filho (maus-tratos, abuso e negligência nos cuidados, superproteção, permissividade, rejeição, pouca interação verbal ou física e interação agressiva) e (b) as ações que os pais têm em resposta às ações de seu filho (uso intenso e consistente de punição física, supervisão e monitoria pobre, pouco reforçamento para comportamentos apropriados, uso de reforçamento de forma inconsistente). Em contrapartida, os comportamentos parentais protetores aos problemas de comportamento seriam: suporte parental - condução afetuosa, com demonstração de aceitação da criança, valorização da criança e apoio às suas iniciativas, encorajamento ao desenvolvimento de competência social, ocorrência frequente de interações mais positivas do que aversivas, expressão frequente de afeto positivo, fortalecimento da identificação com os pais, apoio ao desenvolvimento da autonomia; capacidade de fazer escolhas e promover a autodireção; uso de métodos mais racionais e verbais de disciplina do que os físicos; minimização de brigas e agressão entre os familiares.

De fato, as habilidades implicadas no uso adequado da disciplina, o acompanhamento dos filhos, o uso adequado do reforço positivo, o emprego eficaz das técnicas de solução de problemas ou das habilidades de comunicação estão entre as habilidades que podem colocar ambos os pais fora da zona de risco de fracasso, na educação e nos cuidados dos filhos (Olivares, Mendéz \& Ros, 2005). Diversos pesquisadores apontam para a importância da participação de toda a família nos programas de intervenção, pois a família é responsável pela solução de problemas diários que surgem durante as várias etapas de desenvolvimento de seus filhos 
(Dessen \& Silva, 2004; Guralnick, 1998; Shonkoff \& Meisels, 1999; Williams \& Aiello, 2004).

Sendo assim, os programas de intervenção focados para diminuição dos problemas de comportamento das crianças devem ter como ponto central o trabalho com a família. Por exemplo, Pinheiro, Haase, Del Prette A., Amarante e Del Prette Z. (2006) realizaram um programa de Treinamento de Habilidades Sociais com 34 participantes (entre pais e mães de crianças da 1 à à $4 \underline{a}$ série do Ensino Fundamental que tinham problemas de comportamento). 0 programa foi realizado em 11 encontros semanais, tendo como foco os princípios da análise do comportamento para a prática disciplinar não coerciva e modelos de habilidades sociais educativas parentais; as tarefas semanais eram observar o comportamento do filho e estabelecer condições de aprendizagem e desempenho de comportamentos desejáveis dos filhos. Esses participantes foram avaliados antes e após a intervenção por meio de questionários e entrevistas. Os resultados mostraram uma redução dos comportamentos inoportunos e das situações em que as crianças apresentavam problemas em casa. Os pais e as mães modificaram seus comportamentos com os filhos e foram reforçados positivamente com tal mudança, ao constatarem alterações nos comportamentos-problema das crianças.

Bolsoni-Silva, Del Prette A. e Del Prette Z. (2000) realizaram um programa de Treinamento de Habilidades Sociais com quatro casais, que tinham filhos de dois a 15 anos de idade. 0 programa teve duração de cinco semanas, com dois encontros por semana, sendo avaliado por meio de entrevistas e inventários, aplicados antes e após a intervenção. Após a participação no programa, ambos os pais apontaram: redução nos problemas de relacionamento com os filhos; melhora em habilidades interpessoais; maior concordância entre o casal, com diminuição dos conflitos conjugais; avaliação menos negativa dos comportamentos dos filhos; melhor percepção de aspectos positivos no relacionamento entre pais/mães e filho; estabelecimento de maior número de regras de relacionamento com os filhos (valorizando e identificando a necessidade de: expressar sentimentos positivos e negativos aos filhos, utilizar procedimentos de aproximação gradual para as mudanças de comportamento dos filhos, respeitar os direitos dos filhos, controlar as próprias emoções, prestar atenção seletiva aos comportamentos dos filhos e optar por respostas assertivas em vez de passivas ou agressivas); maior uso de reforçamento positivo, maior habilidade de resolução de problemas; e redução no uso de punições. Além disso, os pais e as mães relataram as seguintes aquisições pessoais: emissão ou tentativas de emissão de comportamentos antecipatórios para amenizar o sentido impositivo de ordens, reações mais adequadas diante dos comportamentos dos filhos, maior expressividade de afeto positivo e harmonia conjugal em relação aos filhos.

Apesar da eficácia de tais programas de intervenção, grande parte das pessoas que participaram era de mães, sendo baixa a participação dos pais (homens). Nota-se a necessidade de os estudos de intervenção envolverem também os pais, considerando, principalmente, que diversos estudos apontam para sua importância no desenvolvimento socioemocional e no desempenho acadêmico dos filhos (Cia, D’Affonseca \& Barham, 2004; Cia \& Barham, 2009; Flouri \& Buchanan, 1997; Lamb, 1997; Lindsey, Caldera \& Tankersley, 2009; Newman, Harrison, Dashiff \& Daviesm, 2008); esses estudos também mostram que a identidade dos pais está cada vez mais voltada para os cuidados e para a interação familiar, não sendo exclusiva da sua formação profissional, como ocorria na geração anterior (Cia \& Barham, 2008). Sendo assim, este estudo teve por objetivo avaliar a eficácia de um programa de intervenção para pais e mães sobre os comportamentos dos filhos.

\section{Metodologia}

\section{Participantes}

Esta fase do estudo contou com a participação de 97 pais e mães (29 do GE1, 34 do GE2 e 34 do GC) de crianças da $1^{\text {a }}(21,2 \%)$ e $2^{\text {a }}(78,8 \%)$ séries do Ensino Fundamental, com média de idade de oito anos. Dentre as crianças, 50 delas eram do sexo feminino e 49 do sexo masculino. A média de idade dos pais era de 35 anos, variando entre 23 e 58 anos e a média de idade das mães era de 32 anos, variando entre 20 e 55 anos. 0 número de filhos foi, em média, dois e todos os participantes eram casados. Em relação à classe socioeconômica, 7,1\% das famílias eram de classe D, 50,5\% eram de classe $C$, $35,4 \%$ eram de classe B2 e 7,1\% eram de classe B1 
(segundo critério Brasil, que tem por objetivo medir o poder aquisitivo do consumidor e classificar a população em cinco classes socioeconômicas). Todos os pais e $97 \%$ das mães exerciam algum tipo de atividade remunerada.

\section{Local da coleta de dados}

A coleta de dados e a intervenção com os pais ocorreram em duas escolas municipais e em uma escola estadual, localizadas em um município no interior do estado de São Paulo.

\section{Medidas avaliativas: Social Skills Rating System (SSRS), versão para pais}

O Social Skills Rating System (SSRS) é um sistema que avalia o repertório de habilidades sociais, os problemas de comportamento e a competência acadêmica de crianças (da Educação Infantil à 6 ${ }^{\text {a }}$ série); o sistema foi elaborado por Gresham e Elliott (1990) e validado para o nosso contexto por Bandeira, Del Prette A., Del Prette Z. e Magalhães (2009). Essa versão do SSRS foi aplicada para avaliar a percepção de pais e mães quanto à existência e intensidade de problemas de comportamento internalizantes e externalizantes nas crianças. 0 sistema foi composto por uma escala tipo Likert com 17 itens em que os pais assinalaram qual a frequência (nunca, algumas vezes e muito frequente) com a qual a criança emite cada um dos comportamentos-problema (sendo distribuídos em três fatores: hiperatividade, comportamentos problemáticos externalizantes e comportamentos problemáticos internalizantes). Por meio da análise de consistência interna, constatou-se que o SSRS-Versão para Pais apresentou $\alpha=0,83$ quando avaliado pelo pai e $\alpha=0,81$ quando avaliado pela mãe.

\section{Procedimento de coleta de dados}

Para separar os efeitos da intervenção de mudanças produzidas por demais variáveis (história, maturação da criança, mudanças de atitudes de ambos os pais e dos professores de acordo com a idade da criança), formaram-se três grupos: experimental 1 (GE1 - formado por homens que participaram da intervenção), experimental 2 (GE2 - formado por homens, cujas esposas participaram da intervenção) e controle (GC - formado por homens que quiseram participar da intervenção, mas foram impedidos por causa dos horários). 0 grupo controle, por uma questão ética, recebeu a intervenção (após passarem pela coleta de dados), mas esta não foi avaliada neste estudo.

Para medir os impactos do programa de intervenção para pais sobre o envolvimento paterno e o desenvolvimento da criança, utilizou-se um delineamento pré/pós-teste com grupo controle não equivalente. Segundo Cozby (2006), nesse delineamento existe grupo experimental e grupo controle, mas os participantes não foram aleatoriamente selecionados, ou seja, foram distribuídos pelas condições. Apesar de haver a possibilidade de os dois grupos não serem equivalentes, os escores do pré-teste podem ser comparados com os escores do pós-teste.

Nos grupos GE1, GE2 e GC, solicitou-se a participação das crianças (de $1^{\underline{a}}$ e $2^{\underline{a}}$ série do Ensino Fundamental), dos seus pais, das suas mães e dos seus professores (existe um professor para cada sala de $1^{\text {a }}$ e $2^{\underline{a}}$ séries, nas instituições-alvo deste estudo). Utilizaram-se como critérios para participantes: a criança viver com ambos os pais (biológicos ou não), a criança estar alfabetizada (considerou-se alfabetizada a criança que apresentou o mínimo de cinco pontos em escrita e aritmética e 14 pontos em leitura no TDE), o pai estar empregado, e se interessar em participar da intervenção. Os pais do GC atenderam a todos esses critérios; eles não participaram da intervenção por não terem horário compatível com os oferecidos para realização do grupo. Considerando os pais que ingressaram nos grupos de intervenção, houve desistência de 14 pais na primeira (58,3\%), sete pais na segunda $(22,6 \%)$ e cinco na terceira escola $(14,7 \%)$. No total, foram entregues para pais e mães aproximadamente 180 convites na primeira escola e 240 na segunda e terceira escolas.

Os participantes que não se enquadravam nesse perfil foram convidados a participar do grupo no ano seguinte. Os três grupos foram constituídos sem a utilização de sorteios. Para analisar o impacto da intervenção a curto prazo, consideraram-se os participantes que permaneceram na pesquisa no pré-teste e pós-teste; e para analisar o impacto da intervenção a longo prazo, consideraram-se os participantes que permaneceram na pesquisa no follow-up. 
Para que os pais e mães dessem seu consentimento para sua própria participação, foram entregues os Termos de Consentimento Livre e Esclarecido (TCLE). A coleta de dados seguiu o mesmo procedimento no pré-teste, no pós-teste e no follow-up (antes, imediatamente após e nove meses após a intervenção com os pais e as mães, respectivamente). Após o consentimento dos pais, o instrumento SSRS-Versão para Pais foi aplicado com os pais e as mães, sob a forma de entrevista (tempo para aplicação de 20 minutos).

\section{Intervenç̃̃o}

A intervenção focou o ensino de práticas parentais favorecedoras de comportamentos pró-acadêmicos por parte dos filhos. Utilizaram-se técnicas cognitivo-comportamentais, tendo por objetivo orientar e trabalhar os participantes sobre: (a) os fundamentos da análise aplicada do comportamento; (b) a necessidade de motivar seus filhos a se comportarem bem e a terem comportamentos adequados aos estudos; (c) a identificação dos determinantes de comportamentos desadaptativos dos filhos; (d) a aplicação, no dia a dia, dos procedimentos básicos de modificação do comportamento e (e) as crenças e os valores dos pais. A intervenção se baseou no pressuposto de que as crianças precisam ser reforçadas de modo frequente, contingente, intenso, diferenciado e sistemático e que precisam ter modelos adequados dos pais, se apoiando em intervenções realizadas por Caballo e Simón (2005), Cooper e Cooper (2005), Del Prette A. e Del Prette Z. (2005), Fagan e Iglesias (1999) e Pinheiro et al. (2006), dentre outros teóricos, que seguem uma abordagem cognitivo-comportamental.

Realizou-se a intervenção com os pais em um período de três meses (12 sessões), com encontros semanais de 90 a 120 minutos de duração. Em todas as sessões, foi solicitada tarefa de casa (exceto na sessão 12), sendo retomada e discutida na próxima sessão. Além disso, durante as sessões eram anotados os exemplos, comentários e os relatos de tarefa de casa dos pais. As anotações eram realizadas ao longo da intervenção, para garantir a maior fidedignidade das mesmas.

Ao final de cada sessão, os pais responderam a um pequeno questionário para avaliar a sessão e foram entregues materiais explicativos (folders, folhetos, exemplos ilustrativos, entre outros) para que levassem para casa e pudessem consultar, quando necessitassem. Ao longo da intervenção, o desempenho dos pais foi avaliado por meio de atividades de "tarefa de casa", em que os mesmos descreveram as interações semanais com os filhos, a fim de avaliar a generalização da intervenção para o ambiente familiar (Caballo \& Simón, 2005; Del Prette A. \& Del Prette Z., 2005). A participação dos pais nas sessões foi de, em média, 94,6\% (variando entre $90,5 \%$ a $100 \%$ de presença).

Durante o programa de intervenção, os pais poderiam solicitar atendimentos individuais com a pesquisadora, em caso de dificuldades que não poderiam ser solucionadas durante os encontros. Além disso, foi estabelecido com os pais que haveria reposição das sessões para aqueles que precisassem faltar, antes de acontecer a próxima sessão (os pais poderiam repor a falta participando num outro horário, porque havia vários grupos paralelos na mesma escola). Ao final de cada sessão, dois pais, em média, recebiam atendimento individual. No total, foram atendidos oito grupos de pais.

\section{Follow-up}

A coleta de follow-up deu início após um ano da coleta de dados do pré-teste. Houve a desistência de 17 pais e mães (sendo dois pais/mães da primeira escola, cinco pais/mães da segunda escola e dez pais/mães da terceira escola). Os motivos pelas desistências foram mudanças de cidade e de instituição de ensino.

\section{Análise de dados}

Com os dados do instrumento SSRS-Versão para Pais foram realizadas análises de medidas de tendência central e dispersão. Para comparar os dados obtidos, entre os três grupos (GE1, GE2 e GC), no pré-teste, pós-teste e follow-up, utilizou-se o ANOVA, MANOVA (usando o software SPSS- for Windows).

\section{Resultados}

Realizaram-se testes qui-quadrado e ANOVA para averiguar diferenças entre os três grupos de 
participantes quanto aos dados sociodemográficos, nos quais não foram encontradas diferenças estatisticamente significativas. Para avaliar o impacto do programa de intervenção a curto e a longo prazo sobre os comportamentos dos filhos, compararam-se os dados do SSRS-Versão para Pais, que avalia a existência e intensidade de hiperatividade, problemas de comportamento internalizantes, problemas de comportamento externalizantes e problemas de comportamento total. As comparações foram feitas, primeiramente, no pré-teste e no pós-teste e, em seguida, no pós-teste e no follow-up, entre o grupo experimental 1, experimental 2 e controle. A Tabela 1 compara as respostas dos pais e das mães, no pré-teste e no pós-teste, quanto às medidas para indicar a apresentação (ou não) de comportamentos problemas pelas crianças.

Para a maioria dos pais e das mães, tanto no pré-teste quanto no pós-teste, as crianças apresentaram problemas de comportamento internalizantes e externalizantes na média ou abaixo da média. No entanto, para pais e mães, as crianças apresentaram índices de hiperatividade acima da média (Bandeira et al., 2009).

Na comparação dos dados do pré-teste entre os três grupos, conforme as respostas das mães, as crianças do GE1 apresentaram médias para comportamentos problemáticos internalizantes significativamente maiores quando comparadas com as crianças do GC $(\mathrm{F}(2 ; 96)=3,46, \mathrm{p}<0,05)$. Na comparação dos dados do pós-teste entre os grupos, não houve diferenças estatisticamente significativas nos índices de problemas de comportamento das crianças, independente do avaliador.

Nas mudanças ocorridas entre o pré-teste e o pós-teste, comparando cada grupo separadamente, observou-se que as crianças do GE1, segundo a avaliação dos pais, apresentaram um índice menor de problemas de comportamento externalizantes $(\mathrm{F}(62,1 ; 28)$ $=79,3, \mathrm{p}<0,001)$, internalizantes $(\mathrm{F}(14,5 ; 28)=13,1$, $\mathrm{p}<0,01)$ e totais $(\mathrm{F}(212,4 ; 28)=41,6, \mathrm{p}<0,001)$ na fase do pós-teste. Considerando as respostas das mães, essas crianças apresentaram um índice de problemas de comportamento externalizantes $(F(70,6$; 28) $=44,3, \mathrm{p}<0,001)$ e totais $(\mathrm{F}(162,2 ; 28)=33,1, \mathrm{p}<$ $0,001)$, significativamente menor na fase de pós-teste, como mostram os dados da Tabela 1.

De forma similar, no GE2, as crianças também apresentaram um índice significativamente menor de problemas de comportamento externalizantes $(F(51,7 ; 35)=37,8, p<0,001$, segundo a avaliação dos pais; $F(55,1 ; 35)=38,3, p<0,001$, segundo a avaliação das mães) e totais $(\mathrm{F}(180,5 ; 35)=33,7$, $\mathrm{p}<0,001$, segundo a avaliação dos pais) na fase de pós-teste.

A Tabela 2 mostra a existência ou não de comportamentos problemas nas crianças, segundo a opinião dos pais e das mães, no pós-teste e no follow-up.

Para a maioria dos pais e das mães, na fase de follow-up, as crianças apresentaram problemas de comportamento total na média ou abaixo da média, considerando a amostra de referência (Bandeira et al., 2009). Na comparação dos dados do follow-up entre os três grupos, as crianças do GE1 apresentaram médias para comportamentos problemáticos externalizantes significativamente maiores, segundo as mães, quando comparadas com as crianças do GC $(\mathrm{F}(2 ; 79)=$ $7,40, p<0,01$ ). Segundo os pais, não houve diferenças estatisticamente significativas nos problemas de comportamento das crianças, na fase de follow-up.

Nas mudanças ocorridas entre o pós-teste e o follow-up, comparando cada grupo separadamente, observou-se que as crianças do GE1 e GE2, segundo a avaliação das mães, apresentaram um índice menor de problemas de comportamento externalizantes $(\mathrm{F}(14,1 ; 23)=14,1, \mathrm{p}<0,01-\mathrm{GE} 1 ; \mathrm{F}(7,11 ; 30)=7,26$, $\mathrm{p}<0,05-\mathrm{GE} 2)$ e totais $(\mathrm{F}(27,1 ; 23)=16,33, \mathrm{p}<0,05$ GE1; $F(27,1 ; 26)=5,24, p<0,05-G E 2)$ na fase do follow-up.

\section{Discussão}

De modo geral, os pais e as mães avaliaram as crianças como tendo problemas de comportamento na média ou abaixo da média. Tal característica se torna importante porque crianças com boa competência social tendem a ter melhor autoconceito, melhor desempenho acadêmico, relacionamentos mais adequados com os pares (D'AvilaBacarji, Marturano \& Elias, 2005; Del Prette A. \& Del Prette Z., 2005; Dessen \& Szelbracikowski, 2004; Dunn, Cheng, O'Connor \& Bridges, 2004; López, 2004), sendo um fator de proteção para possíveis intercorrências ao longo do desenvolvimento (Bongers, Koot, Ende \& Verhulst, 2004; Coley, Morris \& Hernandez, 2004).

O programa de intervenção com os pais mostrou-se promissor, a curto e a longo prazo na: diminuição dos problemas de comportamento externalizantes (segundo pais e mães), internalizantes (segundo pais) 
Tabela 1 - Pontuação média nas medidas de problemas de comportamento das crianças, segundo os pais e as mães, no pré-teste e no pós-teste: Comparaç̃̃o do GEl, GE2 e GC

\begin{tabular}{|c|c|c|c|c|c|c|c|c|c|c|c|c|}
\hline \multirow[t]{4}{*}{ Escalas } & \multicolumn{12}{|c|}{ Grupo } \\
\hline & \multicolumn{4}{|c|}{$\begin{array}{c}\text { Experimental } 1 \\
(n=29)\end{array}$} & \multicolumn{4}{|c|}{$\begin{array}{c}\text { Experimental } 2 \\
(n=36)\end{array}$} & \multicolumn{4}{|c|}{$\begin{array}{l}\text { Controle } \\
(n=34)\end{array}$} \\
\hline & \multirow{2}{*}{$\begin{array}{c}\text { Pré- } \\
\text {-teste } \\
\text { Média } \\
\text { (d.p.) }\end{array}$} & \multirow{2}{*}{$\begin{array}{c}\text { Pós- } \\
\text {-teste } \\
\text { Média } \\
\text { (d.p.) }\end{array}$} & \multicolumn{2}{|c|}{ MANOVA } & \multirow{2}{*}{$\begin{array}{c}\text { Pré- } \\
\text {-teste } \\
\text { Média } \\
\text { (d.p.) }\end{array}$} & \multirow{2}{*}{$\begin{array}{c}\text { Pós- } \\
\text {-teste } \\
\text { Média } \\
\text { (d.p.) }\end{array}$} & \multicolumn{2}{|c|}{ MANOVA } & \multirow{2}{*}{$\begin{array}{c}\begin{array}{c}\text { Pré- } \\
\text {-teste }\end{array} \\
\text { Média } \\
\text { (d.p.) }\end{array}$} & \multirow{2}{*}{$\begin{array}{c}\begin{array}{c}\text { Pós- } \\
\text {-teste }\end{array} \\
\text { Média } \\
\text { (d.p.) }\end{array}$} & \multicolumn{2}{|c|}{ MANOVA } \\
\hline & & & F & gl & & & $\mathbf{F}$ & gl & & & $F$ & gl \\
\hline \multicolumn{13}{|l|}{ Pais } \\
\hline HIP & $\begin{array}{c}5,14 \\
(3,25)\end{array}$ & $\begin{array}{c}4,38 \\
(3,09)\end{array}$ & ns & ns & $\begin{array}{c}4,64 \\
(3,21)\end{array}$ & $\begin{array}{c}3,89 \\
(2,98)\end{array}$ & ns & ns & $\begin{array}{c}3,89 \\
(3,35)\end{array}$ & $\begin{array}{c}3,29 \\
(2,88)\end{array}$ & ns & ns \\
\hline CPI & $\begin{array}{c}3,21 \\
(2,02)\end{array}$ & $\begin{array}{c}2,21 \\
(1,92)\end{array}$ & $13,1^{* *}$ & $1 ; 28$ & $\begin{array}{c}2,53 \\
(2,21)\end{array}$ & $\begin{array}{c}1,81 \\
(2,46)\end{array}$ & ns & ns & $\begin{array}{c}1,97 \\
(1,71)\end{array}$ & $\begin{array}{c}1,74 \\
(1,62)\end{array}$ & ns & ns \\
\hline CPE & $\begin{array}{c}6,17 \\
(3,38)\end{array}$ & $\begin{array}{c}4,10 \\
(3,23)\end{array}$ & $79,3^{* * *}$ & $1 ; 28$ & $\begin{array}{c}5,03 \\
(3,56)\end{array}$ & $\begin{array}{c}3,33 \\
(3,09)\end{array}$ & $37,8^{* * *}$ & $1 ; 35$ & $\begin{array}{c}5,18 \\
(3,35)\end{array}$ & $\begin{array}{c}4,24 \\
(3,51)\end{array}$ & ns & ns \\
\hline $\mathrm{CPT}$ & $\begin{array}{l}14,5 \\
(7,40)\end{array}$ & $\begin{array}{l}10,7 \\
(6,84)\end{array}$ & $41,6^{* * *}$ & $1 ; 28$ & $\begin{array}{l}12,2 \\
(7,82)\end{array}$ & $\begin{array}{c}9,03 \\
(7,02)\end{array}$ & $33,7^{* * *}$ & $1 ; 35$ & $\begin{array}{l}11,0 \\
(7,57)\end{array}$ & $\begin{array}{c}9,26 \\
(6,43)\end{array}$ & ns & ns \\
\hline \multicolumn{13}{|l|}{ Mães } \\
\hline HIP & $\begin{array}{c}5,31 \\
(3,29)\end{array}$ & $\begin{array}{c}5,14 \\
(3,67)\end{array}$ & ns & ns & $\begin{array}{c}4,89 \\
(3,50)\end{array}$ & $\begin{array}{c}4,94 \\
(3,48)\end{array}$ & ns & ns & $\begin{array}{c}3,88 \\
(3,40)\end{array}$ & $\begin{array}{c}4,21 \\
(3,68)\end{array}$ & ns & ns \\
\hline CPI & $\begin{array}{c}3,38 \\
(2,18)\end{array}$ & $\begin{array}{c}2,41 \\
(2,11)\end{array}$ & ns & ns & $\begin{array}{c}2,86 \\
(2,34)\end{array}$ & $\begin{array}{c}2,33 \\
(2,07)\end{array}$ & ns & ns & $\begin{array}{c}2,00 \\
(1,74)\end{array}$ & $\begin{array}{c}1,68 \\
(1,65)\end{array}$ & ns & ns \\
\hline CPE & $\begin{array}{c}5,69 \\
(3,52)\end{array}$ & $\begin{array}{c}3,48 \\
(2,95)\end{array}$ & $44,6^{* * *}$ & $1 ; 28$ & $\begin{array}{c}5,83 \\
(3,42)\end{array}$ & $\begin{array}{c}4,19 \\
(3,12)\end{array}$ & $38,3^{* * *}$ & $1 ; 35$ & $\begin{array}{c}5,32 \\
(3,39)\end{array}$ & $\begin{array}{c}5,06 \\
(3,55)\end{array}$ & ns & ns \\
\hline $\mathrm{CPT}$ & $\begin{array}{l}14,4 \\
(7,48)\end{array}$ & $\begin{array}{l}11,0 \\
(7,30)\end{array}$ & $33,1^{* * *}$ & $1 ; 28$ & $\begin{array}{l}13,6 \\
(7,97)\end{array}$ & $\begin{array}{l}11,5 \\
(6,95)\end{array}$ & ns & ns & $\begin{array}{l}11,2 \\
(7,52)\end{array}$ & $\begin{array}{l}10,9 \\
(7,78)\end{array}$ & ns & ns \\
\hline
\end{tabular}

Legenda: HIP = Hiperatividade (faixa média na amostra de referência para pais/mães = 0,62-3,66); CPI = Comportamentos problemáticos internalizantes (média pais/mães $=2,56-6,26$ ); CPE = Comportamentos problemáticos externalizantes (média pais $/$ mães = 3,53-8,77); CPT = Comportamentos problemáticos totais (média pais/mães = 8,04-19,18); ${ }^{* *}=\mathrm{p}<0,01 ;{ }^{* * *}=\mathrm{p}<0,001 ; \mathrm{ns}=$ não apresenta diferenças significativas.

Fonte: Dados da pesquisa.

e totais (segundo pais e mães) entre as crianças do GE1; diminuição dos problemas de comportamento externalizantes (segundo pais e mães) e totais (pais) entre as crianças do GE2. Em todas as sessões da intervenção, práticas parentais eficazes para lidar com os comportamentos dos filhos foram trabalhadas e o reforço e a generalização do aprendizado possivelmente foram promovidos por meio das lições de casa. Os resultados de outros programas de intervenção com pais também apontaram uma diminuição nos problemas de comportamento das crianças (Bolsoni-Silva et al., 2000; Caballo \& Simón, 2005; Duch, 2005; Fagan \& Iglesias, 1999; Pinheiro et al., 2006). Possivelmente, se fosse realizado um trabalho apenas com as crianças, não seria suficiente, uma vez que as suas ações estão quase sempre relacionadas às ações dos pais, nessa faixa etária (Caballo \& Simón, 2005; Conte, 2001; McMahon, 1999; Olivares et al., 2005; Parke, 2004).
Os impactos positivos do grupo de pais mostram que iniciativas, de caráter preventivo (uma vez que as crianças cujos pais participaram da intervenção, não tinham índices de problemas de comportamento clínico), trazem impactos positivos para o desenvolvimento das crianças, podendo evitar o encaminhamento para os serviços de educação especial, caso frequente no nosso contexto (Pamplin, 2005).

Ressalta-se que alguns fatores contribuíram para esses resultados: (a) o grupo teve uma alta adesão dos pais no programa de intervenção, com poucas ausências durante os encontros; (b) os temas abordados nas sessões foram construídos com base nas necessidades dos pais; e (c) todas as sessões foram avaliadas pelos participantes, sendo possível adequá-las de acordo com suas motivações, além de a pesquisadora poder atentar para a aprendizagem dos pais diante dos conteúdos e das temáticas trabalhadas durante as sessões. 
Tabela 2 - Pontuação média nas medidas de problemas de comportamento das crianças, segundo os pais e as mães, no pós-teste e no follow-up: Comparaç̃õo do GEl, GE2 e GC

\begin{tabular}{|c|c|c|c|c|c|c|c|c|c|c|c|c|}
\hline \multirow[t]{4}{*}{ Escalas } & \multicolumn{12}{|c|}{ Grupo } \\
\hline & \multicolumn{4}{|c|}{$\begin{array}{c}\text { Experimental } 1 \\
(n=24)\end{array}$} & \multicolumn{4}{|c|}{$\begin{array}{c}\text { Experimental } 2 \\
(n=31)\end{array}$} & \multicolumn{4}{|c|}{$\begin{array}{l}\text { Controle } \\
(n=27)\end{array}$} \\
\hline & \multirow{2}{*}{$\begin{array}{c}\text { Pós-teste } \\
\text { Média } \\
\text { (d.p.) }\end{array}$} & \multirow{2}{*}{$\begin{array}{c}\text { Follow-up } \\
\text { Média } \\
\text { (d.p.) }\end{array}$} & \multicolumn{2}{|c|}{ MANOVA } & \multirow{2}{*}{$\begin{array}{c}\text { Pós- } \\
\text {-teste } \\
\text { Média } \\
\text { (d.p.) }\end{array}$} & \multirow{2}{*}{$\begin{array}{c}\text { Follow- } \\
\text {-up } \\
\text { Média } \\
\text { (d.p.) }\end{array}$} & \multicolumn{2}{|c|}{ MANOVA } & \multirow{2}{*}{$\begin{array}{c}\text { Pós- } \\
\text {-teste } \\
\text { Média } \\
\text { (d.p.) }\end{array}$} & \multirow{2}{*}{$\begin{array}{c}\text { Follow- } \\
\text {-up } \\
\text { Média } \\
\text { (d.p.) }\end{array}$} & \multicolumn{2}{|c|}{ MANOVA } \\
\hline & & & $\mathbf{F}$ & gl & & & $\mathbf{F}$ & gl & & & $\mathbf{F}$ & gl \\
\hline \multicolumn{13}{|l|}{ Pais } \\
\hline HIP & $\begin{array}{c}4,42 \\
(2,59)\end{array}$ & $\begin{array}{c}4,29 \\
(2,22)\end{array}$ & ns & ns & $\begin{array}{c}3,71 \\
(2,72)\end{array}$ & $\begin{array}{c}3,97 \\
(2,74)\end{array}$ & ns & ns & $\begin{array}{c}3,19 \\
(2,76)\end{array}$ & $\begin{array}{c}3,15 \\
(2,18)\end{array}$ & ns & ns \\
\hline CPI & $\begin{array}{c}2,13 \\
(1,75)\end{array}$ & $\begin{array}{c}2,46 \\
(2,34)\end{array}$ & ns & ns & $\begin{array}{c}1,74 \\
(2,39)\end{array}$ & $\begin{array}{c}1,73 \\
(1,69)\end{array}$ & ns & ns & $\begin{array}{c}1,78 \\
(1,67)\end{array}$ & $\begin{array}{c}2,11 \\
(1,65)\end{array}$ & ns & ns \\
\hline CPE & $\begin{array}{c}4,13 \\
(2,94)\end{array}$ & $\begin{array}{c}3,83 \\
(2,28)\end{array}$ & ns & ns & $\begin{array}{c}3,03 \\
(2,70)\end{array}$ & $\begin{array}{c}2,71 \\
(2,47)\end{array}$ & ns & ns & $\begin{array}{c}3,85 \\
(3,24)\end{array}$ & $\begin{array}{c}3,78 \\
(2,45)\end{array}$ & ns & ns \\
\hline CPT & $\begin{array}{l}10,7 \\
(5,54)\end{array}$ & $\begin{array}{l}10,6 \\
(4,29)\end{array}$ & ns & ns & $\begin{array}{c}8,52 \\
(6,83)\end{array}$ & $\begin{array}{c}8,42 \\
(4,74)\end{array}$ & ns & ns & $\begin{array}{c}8,81 \\
(6,01)\end{array}$ & $\begin{array}{c}9,04 \\
(4,61)\end{array}$ & ns & ns \\
\hline \multicolumn{13}{|l|}{ Mães } \\
\hline HIP & $\begin{array}{c}4,96 \\
(3,16)\end{array}$ & $\begin{array}{c}4,92 \\
(2,67)\end{array}$ & ns & ns & $\begin{array}{c}4,97 \\
(3,24)\end{array}$ & $\begin{array}{c}4,52 \\
(2,91)\end{array}$ & ns & ns & $\begin{array}{c}4,19 \\
(3,68)\end{array}$ & $\begin{array}{c}4,26 \\
(2,81)\end{array}$ & ns & ns \\
\hline CPI & $\begin{array}{c}2,42 \\
(1,86)\end{array}$ & $\begin{array}{c}2,38 \\
(1,76)\end{array}$ & ns & ns & $\begin{array}{c}2,32 \\
(2,10)\end{array}$ & $\begin{array}{c}2,16 \\
(1,75)\end{array}$ & ns & ns & $\begin{array}{c}1,70 \\
(1,71)\end{array}$ & $\begin{array}{c}1,81 \\
(1,59)\end{array}$ & ns & ns \\
\hline CPE & $\begin{array}{c}3,33 \\
(2,68)\end{array}$ & $\begin{array}{c}2,25 \\
(2,09)\end{array}$ & $14,1^{* *}$ & $1 ; 23$ & $\begin{array}{c}4,03 \\
(2,89)\end{array}$ & $\begin{array}{c}3,35 \\
(2,32)\end{array}$ & $7,26^{*}$ & $1 ; 30$ & $\begin{array}{c}4,93 \\
(3,53)\end{array}$ & $\begin{array}{c}4,96 \\
(3,09)\end{array}$ & ns & ns \\
\hline CPT & $\begin{array}{l}10,7 \\
(5,85)\end{array}$ & $\begin{array}{c}9,54 \\
(4,99)\end{array}$ & $7,72^{*}$ & $1 ; 23$ & $\begin{array}{l}11,3 \\
(6,66)\end{array}$ & $\begin{array}{l}10,0 \\
(5,28)\end{array}$ & $5,24 *$ & $1 ; 30$ & $\begin{array}{l}10,8 \\
(7,61)\end{array}$ & $\begin{array}{l}11,0 \\
(5,18)\end{array}$ & ns & ns \\
\hline
\end{tabular}

Legenda: HIP = Hiperatividade (faixa média na amostra de referência para pais $/$ mães = 0,62-3,66); CPI = Comportamentos problemáticos internalizantes (média pais $/$ mães $=2,56-6,26) ; \mathrm{CPE}=$ Comportamentos problemáticos externalizantes (média pais $/ \mathrm{mães}=$ 3,53-8,77); CPT = Comportamentos problemáticos totais (média pais/mães $=8,04-19,18$ ); ${ }^{*}=\mathrm{p}<0,05$; ${ }^{* *}=\mathrm{p}<0,01$; ns $=$ não apresenta diferenças significativas.

Fonte: Dados da pesquisa.

Retomando a teoria Bioecológica de Bronfenbrenner (1996) e avaliando o impacto da intervenção a curto e a longo prazo, nota-se que, no nível microssistêmico, a intervenção para os pais teve por objetivo interferir na qualidade da interação entre pais e filhos (aumentando a frequência e a qualidade da interação). Como consequência, a intervenção pretendia maximizar os impactos positivos dos processos proximais no desenvolvimento da criança, partindo das interações que elas têm com seus pais no ambiente domiciliar, mas com impactos para interações entre a criança e outras pessoas no seu lar e em outros ambientes, os quais também afetam sua trajetória de desenvolvimento. Dessa forma, melhorias na relação entre pais e filhos podem influenciar nos comportamentos da criança em sala de aula, com os pares e com relação ao estudo, e em casa.
Considerando a importância da qualidade dos processos proximais, supõe-se que os pais que participaram dos grupos de intervenção passaram a interagir com maior frequência com as crianças, em atividades mais estimuladoras, complexas e recíprocas; essas atividades constituem aspectos centrais para que os processos proximais sejam mais efetivos. De tal forma, acredita-se que os pais melhoraram seu desempenho em relação às questões retratadas no grupo e mantiveram essas melhorias no período após a conclusão da intervenção; isso é reconhecido na teoria de Bronfenbrenner (1999) como a influência do tempo, isto é, o efeito cumulativo dos padrões de interações interpessoais com outras pessoas somadas com oportunidades de desenvolvimento que decorrem da qualidade estimuladora dos ambientes nos quais a criança está inserida, ao longo do tempo. 
Considerando uma análise do núcleo do modelo que diz respeito à pessoa em desenvolvimento, no caso a criança, nota-se que, neste estudo, as crianças estavam com seus recursos biológicos intactos, pois não tinham deficiências. Além disso, passaram a apresentar, depois que os pais participaram do programa de intervenção, um melhor repertório social e acadêmico. Essas capacidades (geradoras) contribuem para características pessoais, que provocam reações positivas por parte das diferentes pessoas no ambiente social da criança (pais, outros familiares, professores, colegas, entre outros), maximizando os impactos benéficos de todos os processos proximais nos quais as crianças estão envolvidas (Bronfenbrenner, 1999).

No nível mesossistêmico, a intervenção ocorreu pela ligação entre os dois ambientes nos quais a criança mais convive, ou seja, a família e a escola. Além disso, no nível exossistêmico (em relação às crianças), foi objetivo da intervenção trabalhar o manejo de estresse e a relação entre trabalho e família; isso proporcionou impactos positivos para a vida pessoal dos pais, para o seu convívio familiar e, possivelmente para o seu trabalho, já que o ambiente de trabalho dos pais influencia indiretamente no desenvolvimento da criança. A convivência do grupo possivelmente permitiu a formação de laços entre os pais participantes, se transformando numa fonte de apoio social para os pais, mesmo depois do encerramento do grupo, em momentos quando estes pais se encontraram no ambiente da escola, na hora de levar e buscar os filhos.

No nível macrossitêmico, a realização da intervenção com um número considerável de pais (homens) foi possível porque, no momento histórico atual, existe uma maior aceitação cultural de trabalhos promovendo o envolvimento paterno; e porque existe também uma maior participação das mulheres no mercado de trabalho, dando abertura para que os homens também participem dos cuidados e da educação dos filhos.

Em suma, segundo os princípios da teoria Bioecológica de Bronfenbrenner (1996), o impacto do programa de intervenção a curto e a longo prazo demonstra que as mudanças em relação às pessoas diretamente envolvidas com as crianças (no caso, os pais) e em relação ao ambiente domiciliar (a forma do envolvimento parental) influenciam mutuamente os processos proximais (qualidade das interações pais-filhos). Ao longo do tempo, melhorias nesses processos contribuem para ganhos preciosos na trajetória de desenvolvimento das crianças (Bronfenbrenner, 1996).

Por fim, alguns pontos que contribuíram para a generalização do estudo devem ser destacados. Os comportamentos das crianças foram avaliados por pais e mães, segundo os quais, as crianças tiveram melhoras significativas nos problemas de comportamento, no ambiente familiar, após o programa de intervenção. Os dados sociodemográficos dos participantes não apresentaram diferenças estatisticamente significativas quando comparados entre os três grupos e as três instituições, o que indica que as alterações sobre os comportamentos das crianças são decorrentes da participação dos pais e das mães no programa de intervenção e não da influência de variáveis intervenientes.

\section{Considerações finais}

Em curto e longo prazo, o programa de intervenção para pais mostrou-se eficaz em termos dos impactos sobre os problemas de comportamento das crianças. Dessa forma, um programa de intervenção para pais, tal como foi realizado neste estudo, pode nortear trabalhos de outros pesquisadores e especialistas na área que estão tentando intervir com diferentes amostras populacionais. Os resultados deste estudo alertam para a necessidade de investimento e de políticas públicas que viabilizariam a implementação de um programa de prevenção primária com os pais, como apontado por Murta (2007).

Destaca-se a importância de este estudo ter sido conduzido em três instituições de ensino, com populações diferenciadas, contribuindo para a confiança na generalização dos resultados, pelo menos na região do Brasil no qual ele foi conduzido. No entanto, os dados foram coletados por meio de entrevista, o que pode trazer vieses diferentes aos dados. Estudos futuros poderiam complementar e ampliar a validade desses achados, incluindo dados de observação direta, tanto do repertório dos pais como do repertório dos filhos.

Ressalta-se que os dados do estudo devem ser vistos com cautela, uma vez que a seleção dos participantes não foi aleatória e os grupos de intervenção não eram homogêneos em tamanho ou mesmo no gênero dos participantes. Para garantia da validade interna do estudo, o mesmo deveria ser replicado, utilizando um delineamento experimental. 


\section{Referências}

Bandeira, M., Del Prette, Z. A. P., Del Prette, A., \& Magalhães, T. (2009). Validação das escalas de habilidades sociais, comportamentos problemáticos e competência acadêmica (SSRS-BR) para o ensino fundamental. Psicologia Teoria e Pesquisa, 25(2), 271-282. doi:10.1590/S0102-37722009000200016.

Bolsoni-Silva, A. T., Del Prette, A., \& Del Prette, Z. A. P. (2000). Relacionamento pais-filhos: Um programa de desenvolvimento interpessoal em grupo. Psicologia Escolar e Educacional, 3(3), 203-215.

Bongers, H. L., Koot, H. M., Ende, J. V. D., \& Verhulst, F. C. (2004). Developmental trajectories of externalizing behaviors in childhood and adolescence. Child Development, 75(5), 1523-1537. doi:10.1111/ j.1467-8624.2004.00755.x.

Bronfenbrenner, U. (1996). A ecologia do desenvolvimento humano: Experimentos naturais e planejados. Porto Alegre: Artes Médicas.

Bronfenbrenner, U. (1999). Environments in developmental perspective: Theoretical and operational models. In Y. L Fiedman \& T. D. Wachs (Org.). Capitation and assessment of environment across the life (pp. 3-30). Washington: American Psychological Association.

Caballo, V. E., \& Simón, M. A. (2005). Manual de psicologia clínica e do adolescente - Transtornos específicos. São Paulo: Editora Santos.

Cia, F., \& Barham, E. J. (2009). 0 envolvimento paterno e o desenvolvimento social de crianças iniciando as atividades escolares. Psicologia em Estudo, 14, 67-74. doi:10.1590/S1413-73722009000100009.

Cia, F., \& Barham, E. J. (2008). Trabalho noturno e o novo papel: Uma interface difícil. Estudos de Psicologia, 25(2), 213-233. doi:10.1590/S0103-166X200800 0200006.

Cia, F., D’Affonseca, S. M., \& Barham, E. J. (2004). A relação entre envolvimento paterno e desempenho acadêmico dos filhos. Paidéia: Cadernos de Psicologia e Educação, 14(29), 277-286.

Coley, R. L., Morris, J. E., \& Hernandez, D. (2004). Outof-school care and problem behavior trajectories among low-income adolescents: Individual, family, and neighborhood characteristics as adds risks. Child Development, 73(3), 948-965. doi:10.1111/ j.1467-8624.2004.00716.x.
Conte, F. C. S. (2001). Promovendo a relação entre pais e filhos. In M. Delitti (Org.). Sobre comportamento e cognição (pp. 159-166). Santo André: Esetec.

Cooper, B., \& Cooper, N. (2005). What a difference a parent makes! New York: A focus on father workbook to Accompany.

Cozby, P. C. (2006). Métodos de pesquisa em ciências do comportamento. São Paulo: Editora Atlas.

D’avila-Bacarji, K. M. G., Marturano, E. M., \& Elias, L. C. S. (2005). Suporte parental: Um estudo sobre crianças com queixas escolares. Psicologia em Estudo, 10(1), 107-115. doi:10.1590/S1413-73722005000100013.

Del Prette, Z. A. P., \& Del Prette, A. (2005). Psicologia das habilidades sociais na infância: Teoria e Prática. Petrópolis: Vozes.

Dessen, M. A., \& Silva, N. L. P. (2004). A família e os programas de intervenção: Tendências atuais. In E. G. Mendes, M. A. Almeida \& L. C. A. Williams (Org.). Avanços recentes em Educação Especial. (pp. 179187). São Carlos: EDUFSCar.

Dessen, M. A., \& Szelbracikowski, A. C. (2004). Crianças com problemas de comportamento exteriorizado e a dinâmica familiar. Interação em Psicologia, 8(2), 171-180.

Duch, H. (2005). Redefining parent involvement in Head Start: A two-Generation approach. Early Child Development and Care, 175(1), 23-35. doi:10.1080/0 300443042000206237.

Dunn, J., Cheng, H., O'Connor, T. G., \& Bridges, L. (2004). Children's perspective on their relationships with their nonresident fathers: Influences, outcomes and implications. Journal of Child Psychology and Psychiatry, 45(3), 553-566. doi:10.1111/j.14697610.2004.00245.x.

Fagan, J., \& Iglesias, A. (1999). Father involvement program effects on fathers, father figures, and their Head Start children: A quasi-experimental study. Early Childhood Research Quarterly, 14(2), 243-269. doi:10.1016/S0885-2006(99)00008-3.

Flouri, E., \& Buchanan, A. (2003). The role of father involvement in children's later mental health. Journal of Adolescence, 26, 63-78.

Gresham, F. M., \& Elliott, S. N. (1990). Social skills rating system. Circle Pines, MN: American Guidance Service, Inc. 
Guralnick, M. J. (1998). Effectiveness of early intervention for vulnerable children: A developmental perspective. American Journal of Mental Retardation, 102(4), 319345. doi:10.1352/0895-8017(1998)102<0319:EOEI $\mathrm{FV}>2.0 . \mathrm{CO} ; 2$.

Koller, S. H. (2004). Ecologia do Desenvolvimento Humano - Pesquisa e intervenção no Brasil. São Paulo: Casa do Psicólogo.

Lamb, M. E. (1997). Fathers and child development: An introductory overview and guide. In M. E. Lamb (Org.). The role of the father in child developmental (pp. 1-18). New York: John Wiley \& Sons.

Lindsey, E. W., Caldera, Y. M., \& Tankersley, L. (2009). Marital conflict and the quality of young children's peer play behavior: The mediating and moderating role of parent-child emotional reciprocity and attachment security. Journal of Family Psychology, 23(2), 130-145. doi:10.1037/a0014972.

López, F. (2004). Problemas afetivos e de conduta na sala de aula. In C. Coll, A. Marchesi \& J Palácios (Org.). Desenvolvimento psicológico e educação: Transtornos do desenvolvimento e necessidades educativas especiais. (pp. 113-128). Porto Alegre: Artmed.

McMahon, R. J. (1999). Treinamento de pais. In V. E. Caballo (Org.). Manual de técnicas de terapia e modificação do comportamento (pp. 399-422). São Paulo: Editora Santos.

Murta, S. G. (2007). Programas de prevenção a problemas emocionais e comportamentais em crianças e adolescentes: Lições de três décadas de pesquisa. Psicologia: Reflexão e Crítica, 20(1), 01-08. doi:10.1590/S0102-79722007000100002.

Narvaz, M. G., \& Koller, S. H. O Modelo Bioecológico do Desenvolvimento Humano. In S. Koller (Org.). Ecologia do desenvolvimento humano: Pesquisa e Intervenção no Brasil. (pp. 51-64). São Paulo: Casa do Psicólogo, 2004.

Newman, K., Harrison, L., Dashiff, C., \& Daviesm, S. (2006). Relationships between parenting styles and risk behaviors in adolescent health: An integrative literature review. Revista Latino Americana de Enfermagem, 16(1), 142-150. doi:10.1590/S0104-11692008000100022.
Olivares, J., Méndez, F. X., \& Ros, M. C. (2005). 0 treinamento de pais em contextos clínicos e da saúde. In V. E. Caballo \& M. A. Simón (Org.). Manual de psicologia clínica infantil e do adolescente - Transtornos específicos (pp. 365-385). São Paulo: Editora Santos.

Pacheco, J., Alvarenga, P., Reppold, C., Piccinini, C. A., \& Hutz, C. S. (2005). Estabilidade do comportamento anti-social na transição da infância para a adolescência: Uma perspectiva desenvolvimentista. Psicologia: Reflexão e Crítica, 18(1), 55-61. doi:10.1590/S0102-79722 005000100008

Pamplim, R. (2005). A interface família-escola na inclusão da criança com necessidades educacionais especiais. Dissertação de Mestrado em Educação Especial, Programa de Pós-Graduação em Educação Especial, Universidade Federal de São Carlos, São Carlos.

Parke, R. D. (2004). Development in the family. Annual Reviews Psychology, 55, 365-399. doi:10.1146/annurev.psych.55.090902.141528.

Pinheiro, M. I. S., Haase, V. G., Del Prette, A., Amarante, C. L. D., \& Del Prette, Z. A. P. (2006). Treinamento de habilidades sociais educativas para pais de crianças com problemas de comportamento. Psicologia: Reflexão e Crítica, 19(3), 1-15. doi:10.1590/S0102-79722 006000300009.

Shonkoff, J. P., \& Meisels, S. J. (1999). Early childhood intervention: The evolution of a concept. In S. L. Meisels \& J. P. Shonkoff (Org.). Handbook of early childhood intervention (pp. 3-31). New York: Cambridge University Press.

Williams, L. C. A., \& Aiello, A. L. R. (2004). 0 empoderamento de famílias: 0 que é e como medi-lo. In E. G. Mendes, M. A. Almeida \& L. C. A. Williams (Org.). Avanços recentes em Educação Especial (pp. 197202). São Carlos: EDUFSCar. 